\title{
The Use of Trans Abdominal Ultrasound in Assessment of the Lower Uterine Segment Thickness in Patients with Previous Cesarean Section
}

\author{
Ismael M. Mira, Abdel-Monsef A. Sedek, Ahmed T. Ahmed \\ Department of Obstetrics and Gynecology, Faculty of Medicine, Al-Azhar University,Cairo, Egypt \\ Corresponding author: Ahmed T. Ahmed; Mobile: 01125768883; Email: kingohamada@ gmail.com
}

\begin{abstract}
Introduction: There is a steady increase in the rate of cesarean delivery. In Egypt, Cesarean sections were performed in $38.84 \%$ of deliveries in $2008,37.88 \%$ in $2009,39.08 \%$ in $2010,37.72 \%$ in 2011 and $41.17 \%$ in 2012, repeated cesarean sections (RCS) was the main indication. In making plans for delivery, physicians and patients should consider a woman's chance of a successful VBAC as well as the risk of complications from a trial of labor. Approximately 60-80\% of appropriate candidates who attempt vaginal birth after cesarean (VBAC) will be successful. Aim of the Work: was to determine a cut-off value that can be clinically used to allow a safe vaginal delivery by using abdominal ultrasound to evaluate the thickness of the lower uterine segment in patients with history of previous cesarean section. Patients and Methods: The lower uterine segment thickness was measured by both transabdominal ultrasound in 200 cases, gravidas (37 week - 40 week), 100 cases with previous CS (study group) undergoing repeated CS, and the other 100 cases without any scar in the uterus (control group), then the grade of the scar was assessed intraoperatively. Results: By TAS the best cut- off value was $2.5 \mathrm{~mm}$ and this yielded a sensitivity of $77.3 \%$ and a specificity of $73.5 \%$. At this cut-off value, the positive predictive value was $85 \%$ while the negative predictive was $62.5 \%$ and the accuracy was $76 \%$. Conclusion: Ultrasonographic evaluation permits good assessment of the risk of scar complications intra partum. The lower uterine segment thickness is related to the grade of the scar. The best timing to perform the scan is at late third trimester. A cut-off value of $2.5 \mathrm{~mm}$ by TAS can be safely used with high degree of sensitivity and specificity.
\end{abstract}

Key words: Ultrasound, lower uterine segment, previous cesarean section, VBAC.

\section{Introduction}

There is a steady increase in the rate of cesarean delivery with the exception of the years between 1989 and 1996 when the annual rate of cesarean delivery actually decreased. This decrease was largely due to a significantly increased rate of Vaginal Birth After Caesarean (VBAC) and to a closely mirrored decrease in the primary rate. These trends were short lived, and in 2007, the primary cesarean delivery rate was above 30 percent, whereas VBAC rates dropped to 8.5 percent ${ }^{(\mathbf{1})}$.

In Egypt, a significant rise in cesarean section rate has occurred from 4.6\% in 1992 to $10.3 \%$ in 2000. However, hospital -based cesarean deliveries were much higher in1998 $(13.9 \%)$ and increased to $22.0 \%$ in 2000. Although the cesarean section rate was slightly higher in private hospitals, the rate has also increased consistently in public hospitals ${ }^{(2)}$.

Cesarean sections were performed in $38.84 \%$ of deliveries in $2008,37.88 \%$ in 2009 , $39.08 \%$ in $2010,37.72 \%$ in 2011 and $41.17 \%$ in 2012, repeated cesarean sections (RCS) was the main indication and a large proportion of patients were having previous one cesarean section with an incidence of about $43.5 \%$ in the past 5 years. So, there is an increase in CS rate in Cairo University Hospital and the increase was mainly due to increase in the primary CS rate ${ }^{(3)}$.

In making plans for delivery, physicians and patients should consider a woman's chance of a successful VBAC as well as the risk of complications from a trial of labor. Approximately $60-80 \%$ of appropriate candidates who attempt VBAC will be successful ${ }^{(4)}$.

VBAC avoids major abdominal surgery, lowers a woman's risk of hemorrhage and infection, and shortens postpartum recovery. It may also help women avoid the possible future risks of having multiple cesareans such as hysterectomy, bowel and bladder injury, transfusion and abnormal placenta conditions (placenta previa and placenta accreta) ${ }^{(4)}$.

Among factors that should be considered in selection of candidates for vaginal birth after cesarean delivery (VBAC): one previous prior low-transverse cesarean delivery, clinically adequate pelvis, no other uterine scars or previous 
rupture, physician immediately available throughout active labor capable of monitoring labor and performing an emergency cesarean delivery and availability of anesthesia and personnel for emergency cesarean delivery ${ }^{(1)}$.

A successful VBAC has fewer complications than an elective repeated cesarean while a failed TOLAC has more complications than an elective repeated cesarean ${ }^{(4)}$.

The risk of uterine rupture in laboring women with a previous CS varies between 0.2 and $1.5 \%$ after induction of labor, compared to $0.5 \%$ in women with spontaneous labor after a previous $\mathrm{CS}{ }^{(5)}$.

Uterine rupture requires immediate surgical intervention and its occurrence can result in severe morbidity and mortality for infant and mother. Accurate prediction of uterine rupture would therefore be extremely valuable, as it would allow women at low risk to proceed with a trial of labour (TOL), whereas women at high risk for uterine rupture could undergo a planned $\mathrm{CS}^{(5)}$.

Out of 12 studies, 7 measured the full LUS thickness, 4 measured the myometrial layer only, and 1 measured both. However, owing to the heterogeneity of the studies, no ideal cut-off for lower uterine thickness could be recommended for clinical purposes, and the optimal cut-off value varied from 2.0 to $3.5 \mathrm{~mm}$ for the full LUS

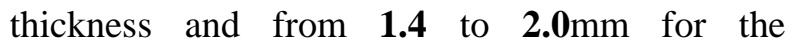
myometrial layer ${ }^{(\boldsymbol{\sigma})}$.

The aim of this study was to determine a cut-off value that can be clinically used to allow a safe vaginal delivery by using abdominal ultrasound to evaluate the thickness of the lower uterine segment in patients with history of previous cesarean section.

\section{Subjects and methods}

This prospective observational study included a total of 200 pregnant women attending at both the outpatient clinic and the inpatient wards of Elsayed Galal, Al-Azhar University Hospital and Helwan General Hospital. Approval of the ethical committee and a written informed consent from all the subjects were obtained. This study was conducted between February 2018 to November 2018

The 200 pregnant women were divided into two groups, each 100: Group A: with previous cesarean section (study group) and Group B: without previous cesarean delivery (control group).

\section{Inclusion criteria:}

1. Pregnant women without any medical disorders.

2. Gestational age: $37-42$ weeks.

3. One previous prior low-transverse cesarean delivery.

4. Clinically adequate pelvis.

5. Normal ultrasonographic findings (gestational age, fetal structures and placental site).

6. Cephalic vertex presentation.

7. Parity: all subjects must have had either: history of previous C.S (study group) or history of normal vaginal delivery (control group). All subjects were informed about the procedure, its value and absence of expected complication.

8. Patients in labour.

Exclusion criteria:

1. Previous repair of ruptured uterus.

2. Prior $\mathrm{T}$-shaped or classic incision.

3. Multiple pregnancies.

4. Previous operations in uterus rather than cs.

5. Polyhydramnios.

6. Previous puerperal sepsis.

7. Oversized fetus.

8. Uterine fibroid.

\section{All participants were subjected to the} following:

1. Thorough history taking (age, gravidity, parity, time interval between previous cesarean sections and current pregnancy, indication for the previous cesarean section, and fetal weight of previous delivery) with special emphasis on menstrual history for proper dating and history of previous pregnancy to verify the criteria for inclusion or exclusion.

2. Full general and abdominal examinations which included:

a. Fundal level to correlate with gestational age known by history.

b. Umbilical grip to verify different inclusion and exclusion criteria.

c. Tenderness and shape of the scar.

3. GE E6 Abdominal ultrasound with a probe of 2-5 MHz and Logiq P3 Abdominal ultrasound with a probe of 1.6-4.6 MHz for full obstetric assessment to confirm gestational age, fetal lie and presentation, placental position and its relation to previous C.S scar. Also, to evaluate the lower segment thickness.

Three layers can be identified by ultrasonography in a well-developed lower uterine segment in a midline section of sagittal view, in a partially filled bladder (waiting for 2 hours after 
the last micturition) as has shown that an over distended bladder could elongate the cervical length by stretching the lower uterine segment. They are as follows from inside outwards:

a) Chorioamniotic membrane with decidualized endometrium.

b) A middle layer of myometrium.

c) Utero-vesical peritoneal reflection juxtaposed to muscularis and mucosa of the bladder.

The lower uterine segment will be visualized in sagittal section in the midline.

\section{Outcome measures:}

All participants were followed up till delivery, the mode of delivery was repeated elective C.S; the Cesarean section scar was assessed intra-operatively.

Intra-operatively C.S scar was assessed to see whether it is intact or there is a scar dehiscence, using a grading system developed by Qureshi et al ${ }^{(7)}$ :

- Grade 1: Well developed lower uterine segment (good scar).

- Grade 2: Thin lower segment, content not visible (good scar).

- Grade 3: Translucent lower segment, content visible (bad scar).

- Grade 4: well-circumscribed defect (bad scar).

\section{Statistical Analysis}

Statistical analysis was performed using Statistical package for social science (SPSS) version 17.

\section{Statistical methods}

The following tests were used:

- Descriptive analysis of the results in the form of percentage distribution for qualitative data (minimum, maximum, mean and standard deviation) calculation for quantitative data.

- Cross tabulation test: For comparison between percentages values (Chi-squared).

- Student t- test: For comparison between means of two groups.

- Fisher's exact test: Used to calculate an exact $\mathrm{P}$-value for a $2 \times 2$ frequency table with small number of expected frequencies, for which the Chi-squared test is not appropriate.

- Sensitivity: Probability that a test result will be positive when the disease is present (true positive rate).

- Specificity: Probability that a test result will be negative when the disease is not present (true negative rate).

- Positive predictive value: Probability that the disease is present when the test is positive.

- Negative predictive value: probability that the disease is not present when the test is negative.

- P: The probability/significance value

- P value $>0.05(\mathrm{NS})$ Not significant

- P value $<\mathbf{0 . 0 5} *$ Significant at 0.05 level

- $\mathbf{P}$ value< $0.01 * *$ Highly significant at 0.01 Level

\section{Results}

Table 1 shows that there were no statistically significant differences regarding age, gravidity, parity or duration since last labor between both groups.

\section{A: Comparison between Cases and Controls (tables 1-3)}

Table 1: Descriptive Statistics

\begin{tabular}{|l|c|c|c|}
\hline & $\begin{array}{c}\text { Group A } \\
\text { (previous CS group) } \\
\mathbf{N = 1 0 0}\end{array}$ & $\begin{array}{c}\text { Group B } \\
\text { (Control group) } \\
\mathbf{N = 1 0 0}\end{array}$ & P value \\
\hline Age (year) & $27.2 \pm 4.9$ & $28.4 \pm 5.9$ & 0.125 NS \\
\hline Gravidity & $3.06 \pm 0.97$ & $3.361 \pm .27$ & 0.062 NS \\
\hline Parity & $1.84 \pm 0.95$ & $2.15 \pm 1.23$ & 0.056 NS \\
\hline $\begin{array}{l}\text { Duration since } \\
\text { last delivery (year) }\end{array}$ & $2.78 \pm 1.46$ & $2.991 \pm .36$ & 0.283 NS \\
\hline No of previous CS & $1.50 \pm .78$ & - & - \\
\hline
\end{tabular}

Fundual level cases ranged from 37 to 38 with a mean of 37.59 SD 0.55 and in controls ranged from 37 to 40 with a mean of 37.82 SD 40 ; showing statistical significant difference $(\mathrm{P}=0.004)$.

Gestational level cases ranged from 37 to 39 with a mean of 37.61 SD 0.55 and in controls ranged from 37 to 41 with a mean of 37.95 SD 0.81 ; showing statistical significant difference $(\mathrm{P}<0.001)$. 
Table 2: Comparison between Cases and Controls regarding fundal level and gestational age

\begin{tabular}{|c|c|c|c|c|c|c|c|}
\hline & & $\mathbf{n}$ & Mean & Std. Deviation & Minimum & Maximum & $P$ value \\
\hline \multirow{2}{*}{$\begin{array}{l}\text { Fundal Level } \\
(\mathrm{cm})\end{array}$} & Cases & 100 & 37.59 & 0.55 & 37.00 & 38.00 & \multirow[t]{2}{*}{$0.004 \mathrm{~S}$} \\
\hline & Controls & 100 & 37.82 & 0.56 & 37.00 & 40.00 & \\
\hline \multirow{2}{*}{$\begin{array}{l}\text { Gestational A } \\
\text { (in ws) (by US) }\end{array}$} & Cases & 100 & 37.61 & 0.55 & 37.00 & 39.00 & \multirow[t]{2}{*}{$<0.001 \mathrm{~S}$} \\
\hline & Controls & 100 & 37.95 & 0.81 & 37.00 & 41.00 & \\
\hline
\end{tabular}

Table 2 shows that both fundal level and gestational age were significantly lower in cases compared to their controls $(\mathrm{p}<0.05)$.

Table 3: Comparison between Both Groups regarding LUS thickness

\begin{tabular}{|c|c|c|c|}
\hline $\begin{array}{c}\text { LUS Thickness } \\
\text { (in mm) }\end{array}$ & $\begin{array}{c}\text { Group A } \\
\text { (previous CS group) }\end{array}$ & $\begin{array}{c}\text { Group B } \\
\text { (control group) }\end{array}$ & P value \\
\hline $\mathrm{Abd} \mathrm{S} / \mathrm{S}$ & $3.01 \pm 1.34$ & $3.30 \pm 1.07$ & $0.093 \mathrm{NS}$ \\
\hline
\end{tabular}

Table 3 shows that there were no statistically significant differences between both groups regarding abdominal U/S LUS thickness ( $\mathrm{p}>0.05$ ).

$B$ : Characteristics of CS scar in Cases

Table 4: Characteristics of Anterior Abd. Wall \&LUS scars in Group A

\begin{tabular}{|l|c|}
\hline A- Ant. abd. wall scars & 20 \\
Keloid & 80 \\
Normal & \\
\hline B- Scar Tenderness & 44 \\
Tender & 56 \\
Not tender & 40 \\
\hline C- LUS Thickness by & 60 \\
TAS 2.5 mm & \\
$>2.5 \mathrm{~mm}$ & \\
\hline D- LUS Scar intraoperative Grading & 30 \\
Grade 1 & 36 \\
Grade 2 & 31 \\
Grade 3 & 3 \\
Grade 4 & \\
\hline
\end{tabular}

Table 4 shows that the shape of LUS skin scar in cases appeared normal in $80 \%$ of subjects and abnormal (keloid) in the remaining 20\%, $44 \%$ of cases had tender scar compared to $56 \%$ with no tenderness, there were 40 cases $(40 \%)$ below $2.5 \mathrm{~mm}$ and 60 cases $(60 \%)$ above $2.5 \mathrm{~mm}$ by TAS and $30 \%$ of scars of cases were of grade $1,36 \%$ of grade $2,31 \%$ of grade 3 and only $3 \%$ of grade 4 .

Intra-operative C.S scar was assessed to see whether it was intact or there was a scar dehiscence, using a grading system developed by Qureshi et al. ${ }^{(7)}$ :

- Grade 1: Well developed lower uterine segment (good scar).

- Grade 2: Thin lower segment, content not visible (good scar).

- Grade 3: Translucent lower segment, content visible (bad scar).

-Grade 4: well-circumscribed defect (bad scar).

C: Predictors and Determinants of scar grading in Cases; Good scar: grade (1\&2) and Bad scar grades (tables 5-6) 
Table 5: Abdominal U/S as predictors of scar grading

\begin{tabular}{|c|c|c|c|c|c|c|c|}
\hline & $\begin{array}{c}\text { Area } \\
\text { Under the } \\
\text { Curve }\end{array}$ & $\begin{array}{c}\text { Best Cut- } \\
\text { off } \\
\text { (in mm) }\end{array}$ & Sensitivity \% & Specificity \% & PPV & NPV & Accuracy \\
\hline $\begin{array}{c}\text { Abdominal U/S LUS } \\
\text { Thickness }\end{array}$ & 0.763 & 2.50 & $77.3 \%$ & $73.5 \%$ & $85.0 \%$ & $62.5 \%$ & $76.0 \%$ \\
\hline
\end{tabular}

Table 5 shows U/S predictors of the quality of scar; grades 1 and 2 were considered good scars and grades 3 and 4 were considered bad scars. In this table, the best cut-off for abdominal U/S scar thickness was $2.50 \mathrm{~mm}$ above which the scar will be considered good scar and below which the scar will consider bad scar (AUC $=0.763$, Sensitivity $77.3 \%$ and Specificity $73.5 \%$, at this cut-off value, the positive predictive value is $85 \%$ while the negative predictive is $62.5 \%$ while the accuracy is $76 \%$ ).

Table 6: Comparison between good and bad scars regarding age, gravidity, parity, number of CS and duration since last CS

\begin{tabular}{|ll|l|c|c|c|}
\hline & & N & Mean & Std. Deviation & P value \\
\hline Age & Good & 66 & 27.17 & \pm 4.84 & 0.837 NS \\
& Bad & 34 & 27.38 & \pm 5.12 & \\
\hline Gravidity & Good & 66 & 3.09 & \pm 0.97 & 0.660 NS \\
& Bad & 34 & 3.00 & \pm 0.98 & \\
\hline Parity & Good & 66 & 1.88 & \pm 0.92 & 0.572 NS \\
& Bad & 34 & 1.76 & \pm 1.02 & \\
\hline Number of CS & Good & 66 & 1.30 & \pm 0.61 & $<0.001 \mathbf{S}$ \\
& Bad & 34 & 1.91 & \pm 0.93 & \\
\hline Duration Since Last SC & Good & 66 & 3.07 & \pm 1.59 & $0.005 \mathbf{S}$ \\
(by years) & $\mathrm{Bad}$ & 34 & 2.21 & \pm 0.97 & \\
\hline
\end{tabular}

Table 6 shows that there were no statistically significant differences between good and bad scars regarding age, gravidity and parity of cases ( $p>0.05)$. On the other hand, it was noticed that cases who had more CS were more likely to have bad scars, cases with shorter durations since last CS were also more likely to have worse scars $(\mathrm{p}<0.05)$.

\section{Discussion}

Cesarean section is one of the most frequent surgical interventions worldwide ${ }^{(\boldsymbol{8})}$. Cesarean section is part of human culture since ancient times and there are tales in western and nonwestern cultures of this procedure resulting in live mothers and offspring even dating as old as Greek mythology. Numerous references to cesarean section appear in ancient Hindu, Egyptian, Greek, Chinese, Roman, and other European folklore ${ }^{(9)}$.

some information has been gained from studies of cesarean section scar healing. It was believed that it heals by regeneration of the muscular fibers and by scar tissue formation. The scar can be composed of fibrous tissue and may be a thin linear scar or a wide one, or it may contain a few regenerated muscle fibers ${ }^{(\mathbf{1 0})}$.

A recent review summarized a lot of studies that showed altered growth factors expression in various in vitro and in vivo pathological scar conditions, such as non-healing ulcers or hypertrophic scar tissue ${ }^{(11)}$.

Collagen deposition, which is the main step in the formation of scaring process, is under the control of growth factors. TGF-B (transforming growth factor B), CTGF (connective tissue growth factor), BFGF (basic fibroblast growth factor), VEGF (vascular endothelial growth factor), TNF$\propto$ (tumor necrosis factor $-\propto$ ) and PDGF (platelet derived growth factor) have been all implicated in scar healing ${ }^{(\mathbf{1 1})}$.

The differences in the biologic behavior of the LUS transverse section scarring process at the time of the first CS may explain a lot of clinical phenotype of LUS in a subsequent pregnancy ${ }^{(\mathbf{1 2})}$. 
The Balanced collagen deposition in the wound area is under growth factor control and also is a key step for good wound healing outcome and tissue function restitution ${ }^{(11)}$.

As well as the last 2 decades in obstetric practice, one of the most important topics of debate is decision making in patients with history of previous cesarean delivery ${ }^{(7)}$. The old dictum of (once cesarean always cesarean) ${ }^{(13)}$ is not longer applicable because of the awareness of the obstetricians about the safety of vaginal birth in a scarred uterus as well as the awareness of greater maternal morbidity and mortality in cesarean birth. Attempted vaginal birth after previous cesarean section (VBAC) remains controversial (14).

The success rate of VBAC is significantly affected by a lot of factors including: indication of previous section, prior vaginal delivery, number of previous section, type of prior uterine incision, inter-delivery interval, and estimated birth weight (15).

The mean age for the studied groups was $27.2 \pm 4.9$ years for the study group and $28.4 \pm 5.9$ years for the control group, with no statistically significant difference between the studied groups as regard age.

There was no statistically significant difference between the two groups as regard the site of the placenta, increased incidence of low placenta in cases with previous CS scar.

The aim of this study was not only to demonstrate a strong positive correlation between the scar thickness as measured by ultrasound in the third trimester but also to determine a cut-off value for this scar thickness that can be clinically used with safety. This cut- off value must yield the best sensitivity and specificity in order not to have many CS for really good scars and in the same time not to jeopardize the life of the mother and the fetus in a useless trial of labor. This naturally has its implication on the diagnosis, prognosis and policy of delivery.

In this study, using the scar thickness in the third trimester and then determining the sensitivity and specificity with a range of cut-off value. By TAS, the best cut- off value is $2.5 \mathrm{~mm}$ and this yielded a sensitivity of $77.3 \%$ and a specificity of $73.5 \%$. At this cut-off value, the positive predictive value was $85 \%$ while the negative predictive is $62.5 \%$ while the accuracy is $76 \%$.
Rosenberg et al. ${ }^{(16)}$ performed the largest ever study on the scar thickness by sonography and he deduced a cut-off value of $3.5 \mathrm{~mm}$ giving a sensitivity of $88.0 \%$, specificity $73.2 \%$, positive predictive value $11.8 \%$ and a negative predictive value $99.3 \%$.

Montanarj et at. ${ }^{(17)}$ have reexamined this cut-off value of $3.5 \mathrm{~mm}$ and found that it provides sensitivity and a specificity of $100 \%$ and $75 \%$ respectively, and positive and negative predictive values of $60.7 \%$ and $100 \%$ respectively.

Sen et al. ${ }^{(18)}$ used a cut-off value of $2.5 \mathrm{~mm}$ and reported sensitivity, specificity, positive and negative predictive values as follows: $90.9 \%$, $84 \%, \quad 71.4 \%$ and $95.5 \%$ using abdominal sonography.

Cheung ${ }^{(15)}$ have chosen $1.5 \mathrm{~mm}$ as the best cut-off value and it yielded a sensitivity of $88.9 \%$, a specificity of $59.5 \%$, a positive predictive value of $32.0 \%$, and a negative predictive value of 96.2 $\%$ in predicting a paper-thin or dehisced LUS.

In this study, the mean scar thickness by transabdominal sonography in the third trimester (at $37-40$ weeks gestation) was $3 \pm 1.3 \mathrm{~mm}$ with a range of $1-6 \mathrm{~mm}$.

Vincent et al. ${ }^{(14)}$ reported a scar thickness in the third trimester of $1.9 \pm 1.4 \mathrm{~mm}$, and higher than the results reported by Cheung et al. (15). Gotoh et al. ${ }^{(19)}$ reported scar thickness in the third trimester of $2.1 \pm 0.7 \mathrm{~mm}$.

The variation in the figures worldwide could reflect many contributing factors. The difference in cesarean section techniques especially in the closure of the uterus and peritoneal closure may be a contributing factor. Also, the healing process which is not yet fully understood may share a part in this dilemma. The type of the ultrasound machine and its resolution and the inter observer error also exist. Lastly, but most importantly is that there is yet, no universal method to measure the scar thickness.

Sonographically, the lower uterine segment appears as a 2-layered structure that contains of the urinary bladder inward, (of the echogenic visceral- parietal reflection, including the muscularis and mucosa of the urinary bladder (the outer layer), and the relatively hypo echoic myometrial layer. Usually at late gestation, the chorioamniotic membrane and the decidualized endometrial layer cannot be seen as layers 
separate from the myometrium. If the fetus is vertex presenting, the presenting part may be sitting against the lower uterine segment, and no amniotic fluid can be seen in between these structures ${ }^{(14)}$. Other studies measure the lower segment thickness from the muscularis and mucosa of the bladder on the outer side to the chorioamniotic membrane inside, hence, depending on a 3-layered pattern (18). Lower uterine segment should not be measured during contraction, and so tocography must be performed to detect asymptomatic uterine contraction. This is because in asymptomatic contraction the lower uterine segment becomes thick; condition described by some authors as pseudo dilatation and may give false measurement ${ }^{(20)}$. We did not use tocography and this may have contributed to the difference in results.

It is evident that all previous studies on this issue have demonstrated a significant relation between scar thickness as measured by ultrasound (regardless of the timing of US) and route of sonography. However, the cut-off value for determining safe vaginal delivery is still a great point of controversy. Several authors have performed many trials and reproduced many values but none was satisfactory and no universal cut-off point could be reached to the moment. Many studies had different results and the cut-off point varied from as low as $1.5 \mathrm{~mm}$ to as high as $4 \mathrm{~mm}$. And again, this is attributable to different techniques of sonographic measurement of the lower segment ${ }^{(\mathbf{1 4})}$.

In this study, by using TAS, about 60 $(60 \%)$ had a scar thickness of above $3 \mathrm{~mm}$ and the remaining 40(40\%), had a scar thickness of below $3 \mathrm{~mm}$.

In this study, 30 cases had scar grade one (well developed lower segment), 36 cases had scar grade two (thin lower segment), 31 cases had scar grade three (translucent lower segment, visible content), and only 3 cases had scar grade four (well- circumscribed defect) intra-operatively. The maternal age failed to show statistically significant relation with the scar thickness by TAS. This is consistent with Asakura et al. (21) who stated that scar thickness and gaping was not related to maternal age, However, the interval from the prior cesarean section had a statistically significant positive correlation with the scar thickness by TAS and, meaning that increased duration form the last delivery ( $>2$ years) is associated with increased thickness of the scar and so decreased risk of rupture uterus, and this is consistent with Shipp et al. ${ }^{(22)}$ who found that for inter delivery intervals up to 18 months, the uterine rupture rates were $2.25 \%$ compared with $1.0-5 \%$ with intervals of 19 months or longer. So they conclude that inter delivery intervals of up to 18 months were associated with increased risk of uterine rupture during trial of labor after Cesarean delivery compared with that for longer inter delivery intervals. The same results were reproduced by Motomura et al. ${ }^{(23)}$.

Also, it was noticed that cases who had more CS were found to have bad scars. At last, it is crucial to remember that the interpretation of any diagnostic test is influenced by the clinical suspicion or pretest probability. For patients with a good scar thickness on ultrasonography and a suspicious history or clinical examination, repeating the ultrasound may be done. Clinical data and experience are two important tools that can never be dispensed It would be wise to perform a repeated cesarean section in any patient on clinical suspicion, even if sonography fails to see evident scar thinning. However, it is to be noted that applying sonographic scar thickness measurement in management of VBAC needs further detailed study.

\section{Conclusion}

\section{From the current study the following can be concluded:}

- Ultrasonographic evaluation permits good assessment of the risk of scar complications intra partum, and could allow safer management of delivery.

The lower uterine segment thickness as measured by transabdominal sonography and is related to the grade of the scar. The best timing to perform the scan is at late third trimester.

- $\quad$ A cut-off value of $2.5 \mathrm{~mm}$ by TAS can be safely used with high degree of sensitivity and specificity.

- Delivery by VBAC doesn't increase fetal and maternal morbidity or mortality.

\section{References}

1. Cunningham FG, Leveno KJ, Bloom ST et al. (2010): Cesarean Delivery and Peripartum Hysterectomy. In: Williams Obstetrics, $23^{\text {rd }}$ Ed, McGraw Hill.

2. Khawaja M, Jurdi R , KabakianKhasholian $T$ (2004): Rising trends in cesarean section rates in Egypt. Birth, 31:1216. 
3. Elkhayat W, Abdel-Moneim $\mathbf{M}$, Mohieldein A, Taha A, Alamin B (2013): Cesarean section rate at a tertiary university hospital in Egypt in five years period (20082012),1-2. Available at: https://www.researchgate.net/publication/31 5667975_A_Three_Year_Retrospective_Stu dy_of_Caesarean_Section_Rate_at_Tanta_U niversity_Hospitals.

4. American College of Obstetricians and Gynecologists (ACOG): Guideline on vaginal birth after cesarean delivery, Guideline, gov, retrieved on 9-2-2010. Available at: https://www.health.qld.gov.au/_data/assets/ pdf file/0022/140836/g-vbac.pdf.

5. Kok N, Wiersma IC, Opmeer BC et al. (2013): Ultrasound. Obstet Gynecol., 42: 132-139.

6. Jastrow N, Chaillet N, Roberge S, Morency AM, Lacasse Y, Bujold E (2010): Sonographic lower uterine segment thickness and risk of uterine scar defect: a systematic review. Obstet Gynecol Can., 32(4):321-7.

7. Qureshi IA, Ullah H, Akram MH et al. (2004): Transvaginal versus transabdominal sonography in the evaluation of pelvic pathology. J Coll physicians Surge., 14(7):390-3.

8. Katz VL, Cefalo RC (1988): History and evaluation of cesarean section. In: Phelan JP. New York: Elsevier Science.

9. American College of Obstetricians and Gynecologists (1999): Vaginal Birth after Previous

CesareanDelivery.https://www.unboundmedi cine.com/.../ACOG_practice_bulletin__Vagi nal_birth_after

10.https://www.researchgate.net/.../315678313 ROLE_OF_THREE_DIMENSIONAL_T RA.

11. Werner S, Grose R (2003): Regulation of wound healing by growth factors \& cytokines. Physiologic Rev., 83:835-70.

12. Pollio F, Staibano S, Mascolo $M$ et al. (2006): Uterine dehiscence in term pregnant patients with one previous cesarean delivery: Growth factor immunoexpression\& collagen content in the scarred lower uterine segment. American J ObstetGynecol., 194:527-34.

13. Craigin E (1916): Conservation in obstetrics, N Y Med J., 1:104.
14. Vincent YTC, Oana CC, Birinder SA (2004): Sonographic evaluation of the lower uterine segment in patients with previos cesarean delivery.J Ultrasound Med., 23:1441-47.

15. Cheung VY (2005): Sonographic measurement of the lower uterine segment thickness in women with previous cesarean section, J Obstet Gynecol Can, 27(7):674-81.

16. Rosenberg $P$, Goffinet $F$, Phillippe $\mathbf{H J}$ (1996): Ultrasonographic measurement of lower uterine segment to assess risk of defects of scarred uterus. Lancet, 347:28184.

17. Montanarj L, Alfei A, Lepadatu C et al. (1999): Transvaginal ultrasonic evaluation of thickness of the section of the uterine wall in previous cesarean section Minerva Gynecol., 51(4):107-12.

18. Sen S, Malik S, Salhan S (2004): Ultrasonographic evaluation of lower uterine segment thickness in patients of previous cesarean section. Int $\mathbf{J}$ Gynecol Obstet., 87(3)215-9.

19. Gotoh $\mathrm{H}$, Masuzaki $\mathrm{H}$, Yoshida $A$ et al. (2000): Predicting incomplete uterine rupture with vaginal sonography during the late second trimester in women with prior cesarean. ObstetGynecol., 95:596-600.

20. Karis JP, Hertzberg BS, (1991): sonographic diagnosis of premature cervical dilatation. Potential pitfall due to lower uterine contraction. J. Ultrasound Med., 10, $83-88$

21. Asakura H, Nakai A, Ishikawa G et al. (2000): prediction of uterine dehiscence by measuring lower uterine segment thickness prior to the onset of labor: evaluation by transvaginal ultrasonography, J Nippon Med Sch., 67(5):352-6.

22. Shipp TD, Zelop CM, Repke JT et al. (2001): inter delivery interval \&risk of symptomatic uterine. ObstetGynecol., 95(1):6 -8.

23. Motomura K, Ganchimeg T, Nagata $\mathrm{C}$ et al. (2017): Incidence and outcomes of uterine rupture among women with prior caesarean section: WHO Multicountry Survey on Maternal and Newborn Health. Scientific reports, 7:44093. 